\title{
Planar and nonplanar ion-acoustic envelope solitary waves in a very dense electron-positron-ion plasma
}

\author{
R. Sabry ${ }^{1,2, a}$, W.M. Moslem ${ }^{1,3}$, and P.K. Shukla ${ }^{1,4-11}$ \\ 1 Institut für Theoretische Physik IV, Fakultät für Physik und Astronomie, Ruhr-Universität Bochum, \\ 44780 Bochum, Germany \\ 2 Theoretical Physics Group, Department of Physics, Faculty of Science, Mansoura University, Damietta Branch, \\ New Damietta 34517, Egypt \\ 3 Department of Physics, Faculty of Science-Port Said, Suez Canal University, Egypt \\ 4 The Nonlinear Physics Centre \& Center for Plasma Science and Astrophysics, Ruhr-Universität Bochum, \\ 44780 Bochum, Germany \\ 5 Department of Physics, Umeå University, 90187 Umeå, Sweden \\ 6 Max-Planck-Institut für extraterrestrische Physik, 85741 Garching, Germany \\ 7 GoLP/Institute of Plasmas and Nuclear Fusion, Instituto Superior Técnico, 1049-001 Lisbon, Portugal \\ 8 CCLRC Centre for Fundamental Physics, Rutherford Appleton Laboratory, Chilton, Didcot, Oxon 0X11 0QX, UK \\ 9 SUPA Department of Physics, University of Strathclyde, Glasgow G 40NG, UK \\ 10 School of Physics, Faculty of Science \& Agriculture, University of Kwazulu-Natal, Durban 4000, South Africa \\ 11 Department of Physics, CITT, Islamabad, Pakistan
}

Received 19 September 2008 / Received in final form 26 November 2008

Published online 9 January 2009 - (C) EDP Sciences, Società Italiana di Fisica, Springer-Verlag 2009

\begin{abstract}
Ion-acoustic envelope solitary waves in a very dense plasma comprised of the electrons, positrons and ions are investigated. For this purpose, the quantum hydrodynamic model and the Poisson equation are used. A modified nonlinear Schrödinger equation is derived by employing the reductive perturbation method. The effects of the quantum correction and of the positron density on the propagation and stability of the envelope solitary waves are examined. The nonplanar (cylindrical/spherical) geometry gives rise to an instability period. The latter cannot exist for planar case and it affected by the quantum parameters, as well as the positron density. The present investigation is relevant to white dwarfs.
\end{abstract}

PACS. 52.27.Cm Multicomponent and negative-ion plasmas - 52.35.Fp Electrostatic waves and oscillations (e.g., ion-acoustic waves) - 52.35.Mw Nonlinear phenomena: waves, wave propagation, and other interactions

\section{1 introduction}

Electron-positron $(e-p)$ pairs exist in the plasma emanating both from the pulsars and from the inner region of the accretion disks surrounding the central black holes in the active galactic nuclei [1]. Such pairs can also exist in the Van Allen radiation belts and near the polar cap of fast rotating neutron stars $[2-5]$, in semiconductor plasmas [6], in intense laser fields [7], in tokamaks [8], as well as in the solar atmosphere [9]. An electron-positron plasma usually behaves as a fully ionized gas consisting of the electrons and positrons. Since many of the astrophysical plasmas contain ions besides the electrons and positrons, it is important to study the behavior of nonlinear wave motions in an electron-positron-ion $(e-p-$ i) plasma. During the last three decades, $e-p$ and $e-p-i$

\footnotetext{
${ }^{a}$ e-mail: sabryphys@yahoo.com; sabry@tp4.rub.de
}

plasmas have attracted significant attention among researchers [10-18]. Linear and nonlinear wave propagation in $e-p$ and $e-p-i$ plasmas have been studied by using different models. For example, Popel et al. [13] investigated ionacoustic solitons in three-component plasmas, whose constituents are the electrons, positrons, and singly charged ions. It is found that the presence of the positron component can result in the reduction of the ion-acoustic amplitude. Nejoh [15] studied the effect of the ion temperature on large-amplitude ion-acoustic waves in an $e-p$ - $i$ plasma. It was found that the ion temperature increases the maximum Mach number and decreases the amplitude of the ion-acoustic solitary wave. Also, the region of the existence of the ion-acoustic solitary waves spreads as the ion temperature decreases. For dense $e-p-i$ plasmas, such as in neutron stars or in white dwarfs, the quantum effects are important because the de Broglie wavelength of the charged carriers (usually $e$ and $p$ due to their small mass) 
is comparable to the dimensions of the plasma system. Therefore, the quantum mechanical effects (e.g. tunneling effect and the holes formation) can play an important role and, of course, the collective behavior of the quantum plasma differs from the traditional plasma [19]. Recently, there has been a growing interest in the investigation of collective processes in quantum plasmas [20-33]. For example, double layers [24], solitons [20,22,23,27], and modulation instability of envelope pulses [29-33] have been investigated in different dense plasma situations.

Motivated by the existence of an $e-p-i$ plasma in astrophysical and space environments, we shall investigate planar and nonplanar (cylindrical and spherical) quantum ion-acoustic (QIA) envelope solitary waves in a three species unmagnetized, collisionless quantum plasma that is composed of the electrons, positrons and ions. The present paper is organized as follows: in Section 2 the basic equations governing the nonlinear dynamics of the QIA envelope solitary waves are presented and a cylindrical/spherical nonlinear Schrödinger equation (NLSE) is derived. In Section 3, the stability analysis and the propagation properties of the QIA envelope solitary waves in planar and nonplanar geometries are discussed. Finally, the results are summarized in Section 4.

\section{Derivation of the modified nlse}

We consider a three species quantum plasma system composed of the electrons, positrons, and ions. The nonlinear dynamics of the QIA waves are governed by the following normalized ion fluid equations

$$
\frac{\partial n}{\partial t}+\frac{1}{r^{\nu}} \frac{\partial}{\partial r}\left(r^{\nu} n u\right)=0
$$

and

$$
\frac{\partial u}{\partial t}+u \frac{\partial u}{\partial r}=-\frac{\partial \varphi}{\partial r}
$$

The electron and positron fluid equations are given by, respectively,

$$
\varphi=-\frac{1}{2}+\frac{n_{e}^{2}}{2}-\frac{H^{2}}{2 \sqrt{n_{e}}} \frac{1}{r^{\nu}} \frac{\partial}{\partial r}\left(r^{\nu} \frac{\partial \sqrt{n_{e}}}{\partial r}\right),
$$

and

$$
\varphi=\frac{\sigma}{2}-\frac{\sigma}{2} n_{p}^{2}+\frac{H^{2}}{2 \sqrt{n_{e}}} \frac{1}{r^{\nu}} \frac{\partial}{\partial r}\left(r^{\nu} \frac{\partial \sqrt{n_{p}}}{\partial r}\right) .
$$

Equations (1)-(4) are coupled with the Poisson equation

$$
\frac{1}{r^{\nu}} \frac{\partial}{\partial r}\left(r^{\nu} \frac{\partial \varphi}{\partial r}\right)=\left(1+\mu_{p}\right) n_{e}-\mu_{p} n_{p}-n
$$

where $\nu=0,1$ and 2 stands for the planar, cylindrical and spherical geometries, respectively. $n$ and $u$ are the ion fluid density and velocity, respectively. Here, $H=\hbar \omega_{p e} / 2 k_{B} T_{F e}$ is the quantum diffraction parameter and $\omega_{p e}=\left(4 \pi n_{0} e^{2} / m_{e}\right)^{1 / 2}$ is the electron plasma frequency. Furthermore, $n_{0}, m_{e}, e, k_{B}$ and $\hbar$ are the equilibrium ion density, the electron mass, the magnitude of the electron charge, the Boltzmann constant and the Planck constant divided by $2 \pi$, respectively. $\mu_{p}=n_{p 0} / n_{0}$ is the ratio of positron equilibrium density $n_{p 0}$ to the ion equilibrium density $n_{0}$, and $\sigma=T_{F p} / T_{F e}$ is the ratio of the positron Fermi temperature $T_{F p}$ to the electron Fermi temperature $T_{F e}$.

In order to investigate the amplitude modulation of QIA envelope solitary waves in a (dense) quantum plasma, we shall employ the standard reductive perturbation multiple scales technique [34]. The independent variables are stretched as $\xi=\epsilon\left(r-v_{g} t\right)$ and $\tau=\epsilon^{2} t$, where $\epsilon$ is a small (real) parameter and $v_{g}$ is the envelope group velocity to be determined later. The dependent variables are expanded as

$$
\boldsymbol{\Gamma}(r, t)=\boldsymbol{\Gamma}_{0}+\sum_{m=1}^{\infty} \epsilon^{m} \sum_{L=-\infty}^{\infty} \boldsymbol{\Gamma}_{L}^{(m)}(\xi, \tau) \exp (i L \theta),
$$

where

$$
\begin{aligned}
\boldsymbol{\Gamma}_{L}^{(m)} & =\left[\begin{array}{llllll}
n_{L}^{(m)} & u_{L}^{(m)} & n_{e_{L}}^{(m)} & n_{p_{L}}(m) & \varphi_{L}^{(m)}
\end{array}\right]^{T}, \\
\boldsymbol{\Gamma}_{L}^{(0)} & =\left[\begin{array}{llllll}
1 & 0 & 1 & 1 & 0
\end{array}\right]^{T}, \quad \theta=k r-\omega t,
\end{aligned}
$$

$k$ and $\omega$ are real variables representing the fundamental (carrier) wavenumber and frequency, respectively. All elements of $\boldsymbol{\Gamma}_{L}^{(m)}$ satisfy the reality condition $\boldsymbol{\Gamma}_{-L}^{(m)}=\boldsymbol{\Gamma}_{L}^{*(m)}$, where the asterisk denotes the complex conjugate. Substituting (6) into the basic equations (1)-(5) and collecting terms of the same powers of $\epsilon$, the first-order $(m=1)$ equations with $L=1$, give

$$
\begin{aligned}
& n_{i_{1}}^{(1)}=\left(k^{2} / \omega^{2}\right) \varphi_{1}^{(1)}, \\
& u_{i_{1}}^{(1)}=(k / \omega) \varphi_{1}^{(1)}, \\
& n_{e_{1}}^{(1)}=4\left(4+H^{2} k^{2}\right)^{-1} \varphi_{1}^{(1)}, \\
& n_{p_{1}}^{(1)}=-4\left(4 \sigma+H^{2} k^{2}\right)^{-1} \varphi_{1}^{(1)},
\end{aligned}
$$

and the linear dispersion relation

$$
\begin{aligned}
& \omega^{2}= \\
& \quad \frac{k^{2}\left(H^{2} k^{2}+4\right)\left(H^{2} k^{2}+4 \sigma\right)}{\left(H^{2} k^{4}+4 k^{2}+4\right)\left(H^{2} k^{2}+4 \sigma\right)+8\left(H^{2} k^{2}+2 \sigma+2\right) \mu_{p}} .
\end{aligned}
$$

The second-order $(m=2)$ reduced equations, with $L=1$, are given by

$$
\begin{gathered}
i \xi\left(-\omega n_{1}^{(1)}+k u_{1}^{(1)}\right)+i \tau v_{g}\left(-\omega n_{1}^{(2)}+k u_{1}^{(2)}\right)= \\
\tau v_{g}^{2} \frac{\partial n_{1}^{(1)}}{\partial \xi}-\tau v_{g} \frac{\partial u_{1}^{(1)}}{\partial \xi} \\
-i \omega u_{1}^{(2)}+i k \varphi_{1}^{(2)}=v_{g} \frac{\partial u_{1}^{(1)}}{\partial \xi}-\frac{\partial \varphi_{1}^{(1)}}{\partial \xi}
\end{gathered}
$$




$$
\begin{gathered}
\xi\left(-k^{2} \varphi_{1}^{(1)}+n_{1}^{(1)}-\left(1+\mu_{p}\right) n_{e_{1}}^{(1)}\right. \\
\left.+\mu_{p} n_{p_{1}}^{(1)}\right)+\tau v_{g}\left(-k^{2} \varphi_{1}^{(2)}+n_{1}^{(2)}-\left(1+\mu_{p}\right) n_{e_{1}}^{(2)}\right. \\
\left.+\mu_{p} n_{p_{1}}^{(2)}\right)=-2 i k \tau v_{g} \frac{\partial \varphi_{1}^{(1)}}{\partial \xi} \\
\xi\left(-\varphi_{1}^{(1)}+\frac{4+H^{2} k^{2}}{4} n_{e_{1}}^{(1)}\right)+\tau v_{g}\left(-\varphi_{1}^{(2)}\right. \\
\left.+\frac{4+H^{2} k^{2}}{4} n_{e_{1}}^{(2)}\right)=\frac{1}{2} i k \tau v_{g} H^{2} \frac{\partial n_{e_{1}}^{(1)}}{\partial \xi}
\end{gathered}
$$

and

$$
\begin{aligned}
& \xi\left(\varphi_{1}^{(1)}+\frac{4 \sigma+H^{2} k^{2}}{4} n_{p_{1}}^{(1)}\right) \\
& \quad+\tau v_{g}\left(\varphi_{1}^{(2)}+\frac{4 \sigma+H^{2} k^{2}}{4} n_{p_{1}}^{(2)}\right)=\frac{1}{2} i k \tau v_{g} H^{2} \frac{\partial n_{p_{1}}^{(1)}}{\partial \xi}
\end{aligned}
$$

Solving the system of equations (9) with the help of (7) yields the second-order quantities with $L=1$ as

$$
\begin{aligned}
& n_{1}^{(2)}=\frac{k}{\omega^{3}}\left[\omega^{2} u_{1}^{(2)}-i\left(\omega-k v_{g}\right) \frac{\partial \varphi_{1}^{(1)}}{\partial \xi}\right], \\
& u_{1}^{(2)}=\frac{1}{\omega^{2}}\left[k \omega \varphi_{1}^{(2)}-i\left(\omega-k v_{g}\right) \frac{\partial \varphi_{1}^{(1)}}{\partial \xi}\right], \\
& n_{e_{1}}^{(2)}=\frac{4}{\left(H^{2} k^{2}+4\right)^{2}}\left[\left(4+H^{2} k^{2}\right) \varphi_{1}^{(2)}+2 i k H^{2} \frac{\partial \varphi_{1}^{(1)}}{\partial \xi}\right],
\end{aligned}
$$

$n_{p_{1}}^{(2)}=-\frac{4}{\left(H^{2} k^{2}+4 \sigma\right)^{2}}\left[\left(4 \sigma+H^{2} k^{2}\right) \varphi_{1}^{(2)}+2 i k H^{2} \frac{\partial \varphi_{1}^{(1)}}{\partial \xi}\right]$,

with the compatibility condition

$$
\begin{aligned}
v_{g}= & \frac{\partial \omega}{\partial k} \\
= & \frac{\omega}{k}\left[1+\omega^{2}\left(-1+\frac{4 H^{2}}{\left(4+H^{2} k^{2}\right)^{2}}\right)\right. \\
& \left.+4 \mu_{p} H^{2} \omega^{2}\left(\frac{1}{\left(4+H^{2} k^{2}\right)^{2}}+\frac{1}{\left(4 \sigma+H^{2} k^{2}\right)^{2}}\right)\right] .
\end{aligned}
$$

Recalling that the compatibility condition (11) is the group velocity of the envelope soliton, which is now dependent on the quantum diffraction effect $H$, as well as the ratio of the positron Fermi temperature-to-the electron Fermi temperature $\sigma$.
The second harmonic modes $(m=L=2)$ arising from the nonlinear self-interaction of the carrier waves are obtained in terms $\left[\varphi_{1}^{(1)}\right]^{2}$ as

$$
\begin{gathered}
n_{2}^{(2)}=\frac{k^{2}}{2 \omega^{4}}\left(3 k^{2}+2 \omega^{2} \Delta_{1}\right)\left[\varphi_{1}^{(1)}\right], \\
u_{2}^{(2)}=\frac{k}{2 \omega^{3}}\left(k^{2}+2 \omega^{2} \Delta_{1}\right)\left[\varphi_{1}^{(1)}\right]^{2}, \\
\varphi_{2}^{(2)}=\Delta_{1}\left[\varphi_{1}^{(1)}\right]^{2},
\end{gathered}
$$

$$
n_{e_{2}}^{(2)}=\frac{\left[-8+2 H^{2} k^{2}+\left(4+H^{2} k^{2}\right)^{2} \Delta_{1}\right]}{\left(1+H^{2} k^{2}\right)\left(4+H^{2} k^{2}\right)^{2}}\left[\varphi_{1}^{(1)}\right]^{2},
$$

and

$$
n_{p_{2}}^{(2)}=\frac{-2}{H^{2} k^{2}+\sigma}\left[\frac{\left(4 \sigma-H^{2} k^{2}\right)}{\left(4 \sigma+H^{2} k^{2}\right)^{2}}+\frac{\Delta_{1}}{2}\right]\left[\varphi_{1}^{(1)}\right]^{2},
$$

where $\Delta_{1}$ is given in the Appendix.

The nonlinear self-interaction of the carrier wave also leads to the creation of a zeroth-order harmonic. Its strength is analytically determined by taking $L=0$ component of the third-order reduced equations, which can be expressed as

$$
\begin{gathered}
n_{0}^{(2)}=\left[1+\frac{(1+\sigma) \mu_{p}}{\sigma}\right] \varphi_{0}^{(2)}+\left[\frac{4\left(1+\mu_{p}\right)\left(-4+H^{2} k^{2}\right)}{\left(4+H^{2} k^{2}\right)^{2}}\right. \\
\left.+\frac{4 \mu_{p}\left(4 \sigma-H^{2} k^{2}\right)}{\sigma\left(4 \sigma+H^{2} k^{2}\right)^{2}}\right]\left|\varphi_{1}^{(1)}\right|^{2}, \quad(13 \mathrm{a}) \\
u_{0}^{(2)}=\frac{-\sigma \omega^{2} \Delta_{2}+k^{2} v_{g}\left[\sigma+\mu_{p}(1+\sigma)\right]}{\left[-\sigma+v_{g}^{2}\left(\sigma+\mu_{p}(1+\sigma)\right)\right] \omega^{2}}\left|\varphi_{1}^{(1)}\right|^{2}, \\
n_{e_{0}}^{(2)}=\varphi_{0}^{(2)}+\frac{4\left(-4+H^{2} k^{2}\right)}{\left(4+H^{2} k^{2}\right)^{2}}\left|\varphi_{1}^{(1)}\right|^{2}, \\
n_{p_{0}}^{(2)}=-\varphi_{0}^{(2)}-\frac{4\left(4 \sigma-H^{2} k^{2}\right)}{\left(4 \sigma+H^{2} k^{2}\right)^{2}}\left|\varphi_{1}^{(1)}\right|^{2},
\end{gathered}
$$

and

$$
\varphi_{0}^{(2)}=\frac{\sigma\left(k^{2}-\omega^{2} v_{g} \Delta_{2}\right)}{\left[-\sigma+v_{g}^{2}\left(\sigma+\mu_{p}(1+\sigma)\right)\right] \omega^{2}}\left|\varphi_{1}^{(1)}\right|^{2},
$$

where $\Delta_{2}$ is given in the Appendix.

Finally, the third harmonic modes $(m=3$ and $L=1)$ give a system of equations, which reduces to, with the aid of (11), the modified NLSE

$$
i \frac{\partial \phi}{\partial \tau}+P \frac{\partial^{2} \phi}{\partial \xi^{2}}+Q|\phi|^{2} \phi+i \frac{\nu}{2 \tau} \phi=0,
$$

where $\phi \equiv \varphi_{1}^{(1)}$ for simplicity. The dispersion coefficient $P$ and the nonlinear coefficient $Q$ are given in the Appendix. 


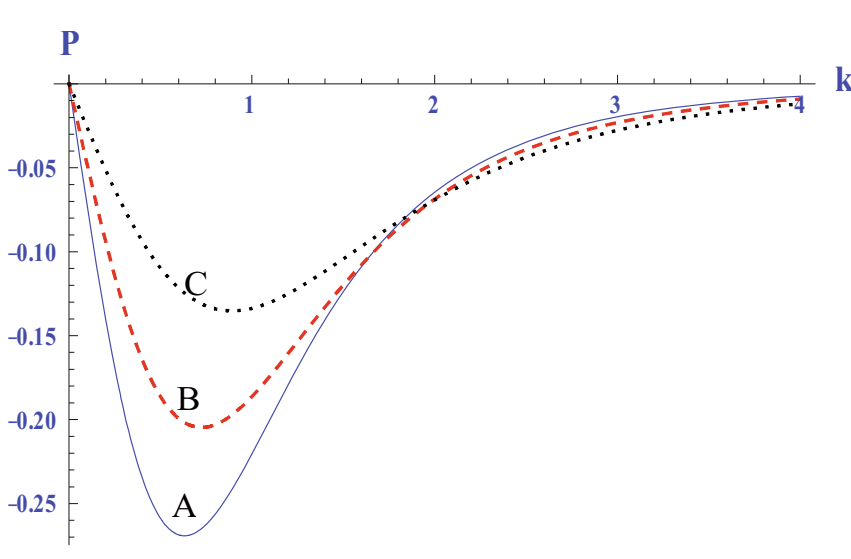

(a)

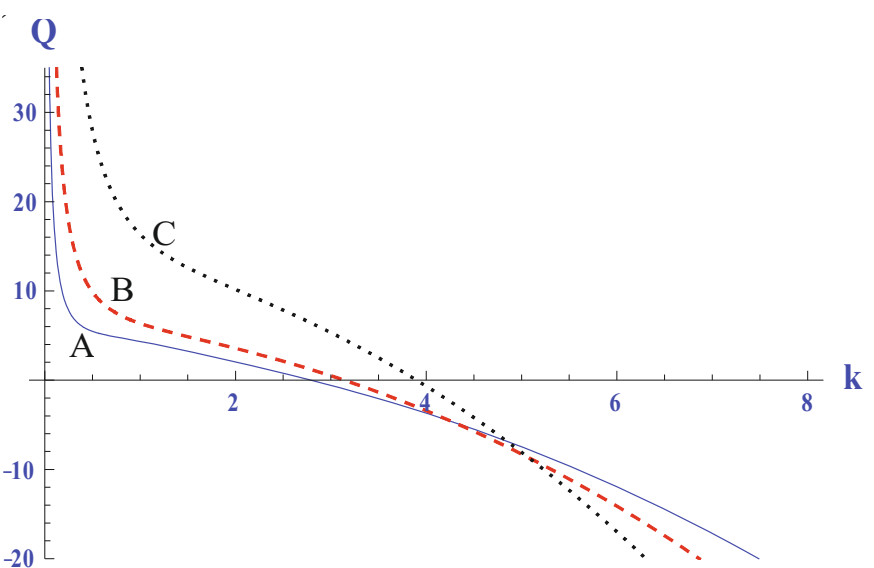

(b)

Fig. 1. (Color online) Variation of (a) $P$ and (b) $Q$ against $k$ for different values of $\mu_{p}$ and $\sigma$. Curve A for $\mu_{p}=0.1$, curve $\mathrm{B}$ for $\mu_{p}=0.3$ and curve $\mathrm{C}$ for $\mu_{p}=0.8$. Recall that $\sigma$ is related to $\mu_{p}$ through the relation $T_{F p} / T_{F e}=\left[\mu_{p} /\left(1+\mu_{p}\right)\right]^{2 / 3}$. Here $H=0.02$.

\section{Stability analysis and discussion}

\subsection{Derivation of the nonlinear dispersion relation}

To investigate the stability/instability of the planar and nonplanar excitations, we investigate the development of the small modulation $\delta \phi$ according to

$$
\phi=\left[\bar{\phi}_{0}+\delta \phi(\xi, \tau)\right] \exp \left[-i \int_{\tau_{0}}^{\tau} \Delta\left(\tau^{\prime}\right) d \tau^{\prime}-\frac{\nu}{2} \ln \tau\right]
$$

where $\bar{\phi}_{0}$ is the constant (real) amplitude of the pump carrier wave and $\Delta$ is a nonlinear frequency shift, and taking the perturbation $\delta \phi$ as

$$
\delta \phi=\delta \bar{\phi}_{0} \exp \left[i\left(K \xi-\int_{\tau_{0}}^{\tau} \Omega\left(\tau^{\prime}\right) d \tau^{\prime}\right)\right]+c . c .,
$$

where $K \xi-\int_{\tau_{0}}^{\tau} \Omega d \tau^{\prime}$ is the modulation phase with $K$ and $\Omega$ are the perturbation wave number and frequency of the modulation, respectively (see details in Refs. $[35,36]$ ). Using (15) and (16) into equation (14), one obtains the nonlinear dispersion relation [35]

$$
\Omega^{2}=\left(P K^{2}\right)^{2}\left(1-\frac{Q}{P} \frac{2\left|\bar{\phi}_{0}\right|^{2}}{\tau^{\nu}} \frac{1}{K^{2}}\right),
$$

which exactly reduces to the dispersion relation for the planar geometry when $\nu=0$.

\subsection{Stability/instability of planar envelope pulse}

To investigate the stability/instability of the planar envelope pulses, one sets $\nu=0$ into the nonlinear dispersion relation (17). It is clear that for $P Q>0$ the envelope is unstable for $K<K_{c}=\sqrt{2 Q / P}\left|\bar{\phi}_{0}\right|$, i.e. for perturbation wavelengths larger than a critical value $2 \pi / K_{c}$. If $P Q<0$, the amplitude will be stable to external perturbations. This modulation instability mechanism is tantamount to the well-known Benjamin-Feir instability in hydrodynamics. Furthermore, the modulation instability is related to the occurrence of localized envelope structures (solitons) of various kinds [37-39]. For the unstable wave packet $(P Q>0)$, it can be shown that the QIA solitary waves propagate as an envelope soliton. On the other hand, for stable wave packet $(P Q<0)$, the wave can propagate in the form of an envelope hole called a dark soliton. When $P Q>0$, for example, one can obtain

$$
\phi(\xi, \tau)=\sqrt{\left|\frac{2 \Theta}{Q}\right|} \operatorname{sech}\left(\sqrt{\left|\frac{2 \Theta}{P}\right|} \xi\right) \exp (i \Theta \tau)
$$

where $\Theta$ is a real constant. It is obvious from equation (18) that the amplitude and the width of the soliton vary with $Q$ and $P$. The wave amplitude is inversely proportional to $|Q|$, and the wave width is proportional to $|P|$. For an extensive list of a number of envelope soliton solutions of the NLSE of the bright or dark (black/grey) type, the reader may consult references $[38,39]$.

We have chosen our physical parameters to be applicable for white dwarfs. Figure 1 shows the variation of $P$ and $Q$ against $k$ for $H=0.02$ and different values of $\mu_{p}$ and $\sigma$. Firstly, we have to confirm the inequality $P Q>0$. Figure 1 should be used on the same footing with Figure 2a; for $H=0.02$, by increasing the positron concentration $\mu_{p}$ and the temperature ratio $\sigma$, one encounter a decrease of the soliton width and amplitude. It is clear also that the effect of $\mu_{p}$ on the soliton amplitude and width is significant for longer wavelengths. Figure 2a shows a contour plot of $P Q$ against $k$ and $H$ for $\mu_{p}=0.1$ and $\sigma=0.20218$. 


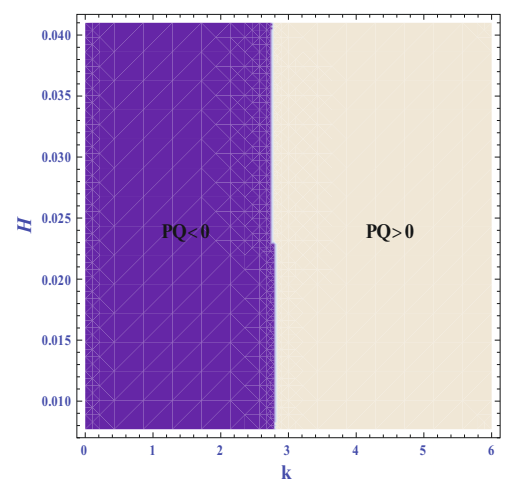

(a)

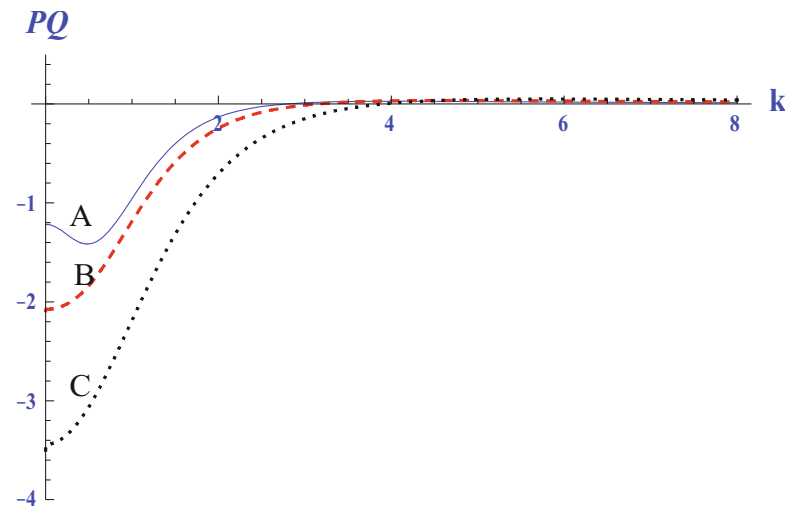

(b)

Fig. 2. (Color online) (a) Variation of $P Q$ in the parameter space $(k, H)$, where bright regions correspond to $P Q>0$ (instability regions) and dark regions corresponds to $P Q<0$ (stability regions). (b) Variation of $P Q$ against $k$ with the same parameters as in Figure 2.

It is obvious that the quantum diffraction effect $H$ has little effect on the instability/stability region and the QIA waves will be unstable for $k>2.8$. Also, increasing the positron concentration increases (decreases) the stability (instability) regions, as depicted in Figure 2b.

\subsection{Stability/instability of spherical and cylindrical pulses}

For $\nu \neq 0$ the nonlinear dispersion relation (17) provides us the instability period for the cylindrical geometry $(\nu=1)$

$$
\tau \geq \tau_{\max }=\frac{2\left|\bar{\phi}_{0}\right|^{2}}{K^{2}} \frac{Q}{P}
$$

and for the spherical geometry $(\nu=2)$

$$
\tau \geq \tau_{\max }=\frac{\left|\bar{\phi}_{0}\right|}{K} \sqrt{\frac{2 Q}{P}} .
$$

It is clear that there is a modulation instability period $(\tau)$ for the cylindrical and spherical wave modulation, which does not exist in the one-dimensional case.

To examine the cylindrical and spherical geometry effects on the propagation of QIA envelope solitary waves, equation (14) can be simplified to

$$
i \frac{\partial \phi}{\partial \tau}+\frac{\partial^{2} \phi}{\partial \xi^{2}}+2|\phi|^{2} \phi+i \frac{\nu}{2 \tau} \phi=0,
$$

where we have set $\phi \rightarrow \sqrt{\frac{2}{Q}} \phi$ and $\xi \rightarrow \sqrt{P} \xi$ with the conditions that $P>0$ and $Q>0$. The stationary propagation of the envelope soliton governed by equation (21) with $\nu=0$ (i.e. the one-dimensional geometry), has the following general form

$$
\begin{aligned}
\phi(\xi, \tau)=A \operatorname{sech} & \left(A \xi-2 A B \tau+c_{0}\right) \\
\times & \exp \left[i B \xi+i\left(A^{2}-B^{2}\right) \tau+i c_{1}\right],
\end{aligned}
$$

where $A, B, c_{0}$, and $c_{1}$ are arbitrary real constants. Solving equation (21) numerically for the cylindrical and spherical geometries; where the initial solution was taken to be of the form (22) with $A=0.11$ and $B=0$. It is found that the amplitude of the QIA envelope solitary waves in the spherical geometry is larger than that in the cylindrical geometry for fixed time, as shown in Figure 3a. Figures $3 \mathrm{~b}-3 \mathrm{~d}$ display the envelope electrostatic potential excitations in three-dimensional plots versus the radial and time coordinates for $\nu=0,1$ and 2 , respectively. It is clear that the envelope soliton pulse for the cylindrical geometry is smaller than the spherical geometry case.

\section{Summary}

We have investigated the amplitude modulation of the planar, cylindrical, and spherical quantum ion-acoustic envelope solitary waves in an unmagnetized quantum plasma consisting of the electrons, positrons, and ions. The standard reductive perturbation method is used to derive a modified NLSE from the quantum hydrodynamic and Poisson equations. It is found that the nature of the modulation instabilities would be significantly modified due to the positron density concentration. Also, there exist a modulation instability period for the cylindrical and spherical envelope excitations, which does not exist in the one-dimensional case. The instability period depends on the positron density concentrations and the quantum diffraction effect. Numerical investigation reveals that the amplitude of the spherical envelope is larger than that of the cylindrical one for fixed time, and the growing amplitude of the spherical envelope is larger than that of the cylindrical envelope. 


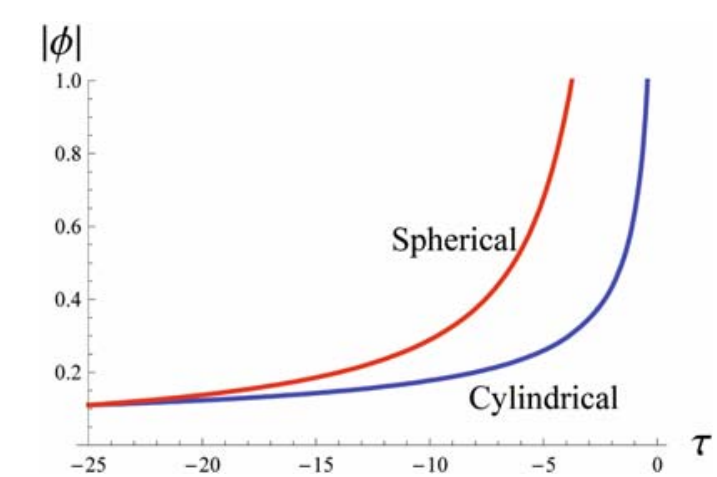

(a)

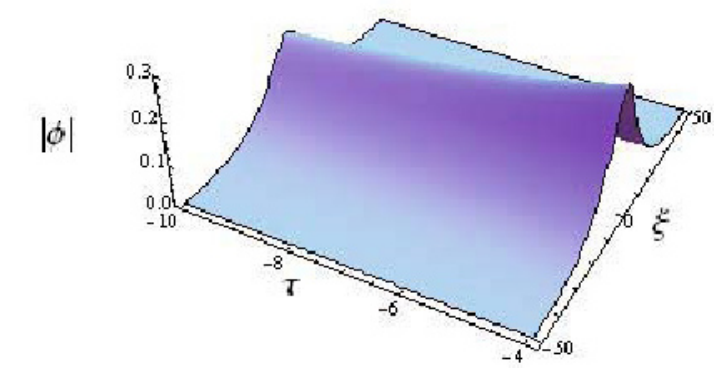

(c) (b)
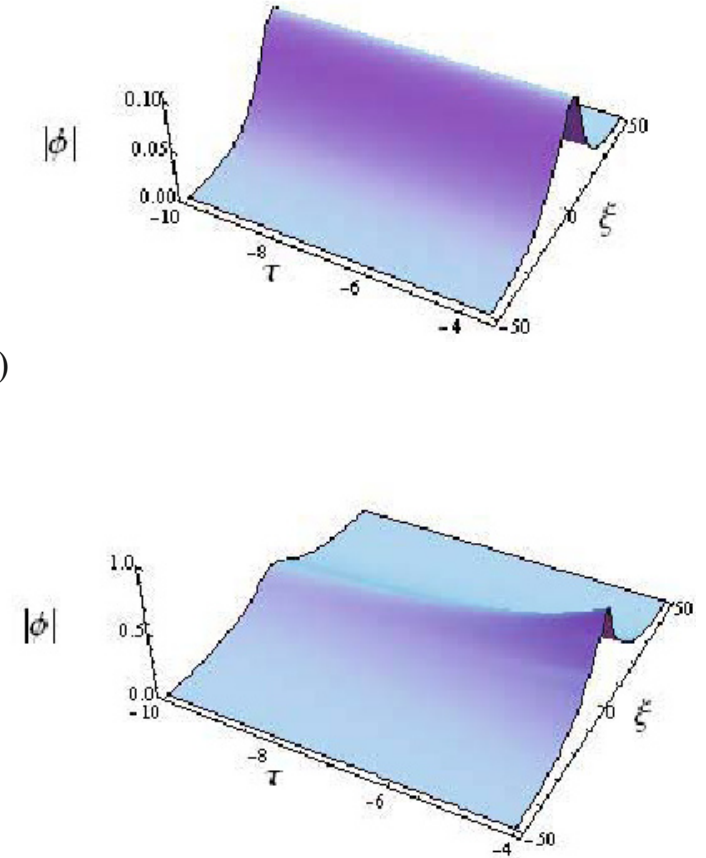

(d)

Fig. 3. (Color online) (a) Cylindrical and spherical waves amplitude against $\tau$ (represented by the numerical solution of the NLSE (21)). (b)-(d) Three dimensional plots of the planar, cylindrical, and spherical envelope pulses, respectively (represented by the numerical solution of the NLSE (21)).

\section{Appendix: Dispersion and nonlinear coefficients}

The dispersion coefficient $P=\frac{1}{2} \frac{\partial^{2} \omega}{\partial k^{2}}$ reads

$$
\begin{array}{r}
P=\left(-\frac{2 v_{g}}{k}+\frac{3 k v_{g}^{2}}{2 \omega}+\frac{\omega}{2 k^{2}}-\frac{\omega^{3}}{2 k^{2}}+\frac{H^{2} \omega^{3}\left(4-3 H^{2} k^{2}\right)}{2 k^{2}\left(4+H^{2} k^{2}\right)^{3}}\right) \\
-\frac{2 \mu_{p} \omega^{3} H^{2}}{k^{2}}\left(\frac{4 H^{2} k^{2}}{\left(4 \sigma+H^{2} k^{2}\right)^{3}}\right. \\
\left.-\frac{4-3 H^{2} k^{2}}{\left(4+H^{2} k^{2}\right)^{3}}-\frac{1}{\left(4 \sigma+H^{2} k^{2}\right)^{2}}\right)
\end{array}
$$

and the nonlinear coefficient $Q$ is

$$
\begin{aligned}
Q=-\frac{\omega^{3}}{4 k^{2} v_{g}}\left(\Delta_{3}-\frac{v_{g}}{\left(1+H^{2} k^{2}\right)\left(4+H^{2} k^{2}\right)^{4}}\right. & \Delta_{4} \\
& \left.+\Delta_{5}+\Delta_{6}\right),
\end{aligned}
$$

where $\Delta_{j}$ (i.e., $j=1,2, \ldots$ )

$$
\Delta_{1}=\frac{\beta_{1}}{\beta_{2}},
$$

$$
\begin{aligned}
\Delta_{5}=\frac{4 \sigma k^{2}\left(8-H^{2} k^{2}\right)}{v_{g}(1+\sigma) \omega^{2}\left(4+H^{2} k^{2}\right)^{2}}\left[1-\frac{\Delta_{2} v_{g}}{k^{2}} \omega^{2}\right] \\
+\frac{4 \Delta_{2}\left(8 \sigma-H^{2} k^{2}\right)}{(\sigma+1)\left(4 \sigma+H^{2} k^{2}\right)^{2}}\left[1-\frac{k^{2}}{\Delta_{2} v_{g} \omega^{2}}\right],
\end{aligned}
$$

$$
\begin{gathered}
\Delta_{2}=-\frac{2 k^{3}}{\omega^{3}}+\frac{4 v_{g}\left[\mu_{p} \beta_{3}-\sigma\left(4-H^{2} k^{2}\right)\left(4 \sigma+H^{2} k^{2}\right)^{2}\right]}{\sigma\left(4+H^{2} k^{2}\right)^{2}\left(4 \sigma+H^{2} k^{2}\right)^{2}}, \\
\Delta_{3}=\frac{5 v_{g} k^{6}}{\omega^{6}}\left[1+\frac{4 \omega}{5 k v_{g}}+\frac{6 \Delta_{1} \omega^{2}}{5 k^{2}}+\frac{2 \omega^{2}}{5 k^{2} v_{g}^{2}}\right. \\
\left.-\frac{2 \Delta_{2} \omega^{4}}{5 k^{4} v_{g}}+\frac{8 \omega^{4}\left(-4+H^{2} k^{2}\right)}{5 k^{4}\left(4+H^{2} k^{2}\right)^{2}}\right], \\
\Delta_{4}=1-2 \Delta_{1}-\frac{H^{2} k^{2}}{8}\left(7-\frac{5}{4} H^{4} k^{4}\right) \\
+\frac{\Delta_{1} H^{2} k^{2}}{4}\left(1+2 H^{2} k^{2}+\frac{5}{16} H^{4} k^{4}\right),
\end{gathered}
$$

and

$$
\Delta_{6}=-\frac{512 v_{g}}{\left(H^{2} k^{2}+4\right)^{4}}\left[1-\frac{3 H^{2} k^{2}}{8}\left(1-\frac{H^{2} k^{2}}{12}\right)\right]
$$

$$
+4 v_{g} \mu_{p} \beta_{7}+\beta_{8}
$$


with $\beta_{j}$ (i.e., $j=1,2, \ldots$ )

$$
\begin{aligned}
& \beta_{1}=-\frac{3 k^{4}}{\omega^{4}}+\frac{4\left(1+\mu_{p}\right)\left(-4+H^{2} k^{2}\right)}{\left(1+H^{2} k^{2}\right)\left(4+H^{2} k^{2}\right)^{2}}-\frac{4 \mu_{p}\left(-4 \sigma+H^{2} k^{2}\right)}{\left(\sigma+H^{2} k^{2}\right)\left(4 \sigma+H^{2} k^{2}\right)^{2}}, \\
& \beta_{2}=\frac{2 k^{2}}{\omega^{2}}\left(1-4 \omega^{2}\right)-2 \mu_{p}\left[\frac{1}{H^{2} k^{2}+\sigma}+\frac{\mu_{p}+1}{\mu_{p}\left(H^{2} k^{2}+1\right)}\right] \\
& \beta_{3}=(\sigma-1)\left[H^{6} k^{6}+8(1+\sigma)\left(-8 \sigma+H^{4} k^{4}\right)+16 H^{2} k^{2}(1+\sigma(\sigma-1))\right], \\
& \beta_{4}=\frac{k^{2}}{\omega^{2}\left(H^{2} k^{2}+4\right)^{2}}\left[1+\frac{24 \omega^{2}}{k^{2}\left(H^{2} k^{2}+4\right)^{2}}-\frac{2 H^{2} \omega^{2}}{\left(H^{2} k^{2}+4\right)^{2}}\right]-\frac{k^{2}}{\sigma \omega^{2}\left(H^{2} k^{2}+4 \sigma\right)^{2}}\left[1-\frac{24 \sigma \omega^{2}}{k^{2}\left(H^{2} k^{2}+4 \sigma\right)^{2}}+\frac{2 \omega^{2}}{k^{2}\left(H^{2} k^{2}+4 \sigma\right)^{2}}\right] \\
& +\frac{28 \sigma}{\left(H^{2} k^{2}+\sigma\right)\left(H^{2} k^{2}+4 \sigma\right)^{4}}\left(1-\frac{5 H^{2} k^{2}}{28 \sigma}\right)-\frac{36}{\left(H^{2} k^{2}+1\right)\left(H^{2} k^{2}+4\right)^{4}}\left(1+\frac{5 H^{2} k^{2}}{36}\right), \\
& \beta_{5}=-\frac{4 k^{2}}{\left(H^{2} k^{2}+4\right)^{2} \omega^{2}}\left[1+\frac{24 \omega^{2}}{k^{2}\left(H^{2} k^{2}+1\right)\left(H^{2} k^{2}+4\right)^{2}}\right]+\frac{4 k^{2}}{\omega^{2}\left(4 \sigma+H^{2} k^{2}\right)^{2}} \times\left[1-\frac{\sigma \omega^{2}}{4 k^{2}\left(4 \sigma+H^{2} k^{2}\right)^{2}}-\frac{\sigma^{2} \omega^{2}}{8 k^{2}\left(4 \sigma+H^{2} k^{2}\right)^{3}}\right], \\
& \beta_{6}=\frac{\left[53(\sigma-1) H^{6} k^{6}+120\left(\sigma^{2}-1\right) H^{4} k^{4}-16\left(5+12 \sigma(\sigma-1)-5 \sigma^{3}\right) H^{2} k^{2}-128 \sigma\left(\sigma^{2}-1\right)\right] \Delta_{1}}{\left(1+H^{2} k^{2}\right)\left(\sigma+H^{2} k^{2}\right)\left(4+H^{2} k^{2}\right)^{2}\left(4 \sigma+H^{2} k^{2}\right)^{2}}, \\
& \beta_{7}=2 \beta_{5}-\beta_{6}+2 H^{2} k^{2} \beta_{4}, \\
& \beta_{8}=\frac{2 \sigma\left(k^{2}-\omega^{2} v_{g} \Delta_{2}\right)\left[\beta_{9}-\beta_{10}+\omega\left(\beta_{11}+\beta_{12}+\beta_{13}+256 \sigma(\sigma+1)\left(\sigma k^{2}+(\sigma-1) \omega^{2}\right)\right)\right]}{(1+\sigma)\left(4+H^{2} k^{2}\right)^{2}\left(4 \sigma+H^{2} k^{2}\right)^{2}\left[\sigma v_{g}^{2}+(\sigma+1) \mu_{p} v_{g}^{2}-\sigma\right] \omega^{5} v_{g}}, \\
& \beta_{9}=2 v_{g} k^{3}(\sigma+1)\left[\left(4+H^{2} k^{2}\right)\left(4 \sigma+H^{2} k^{2}\right)\right]^{2}, \\
& \beta_{10}=4 v_{g}^{2} \omega^{3}\left[H^{6} k^{6}-64 \sigma(1+\sigma)+8 H^{2} k^{2}\left(1-8 \sigma+\sigma^{2}\right)\right], \\
& \beta_{11}=(1+\sigma) H^{8} k^{10}+2\left[4 k^{2}(1+\sigma)^{2}+\omega^{2}(1-\sigma)\right] H^{6} k^{6}, \\
& \beta_{12}=16(1+\sigma)\left[k^{2}\left(1+4 \sigma+\sigma^{2}\right)+\omega^{2}(1-\sigma)\right] H^{4} k^{4} \\
& \beta_{13}=32\left[4 \sigma k^{2}(\sigma+1)^{2}+\omega^{2}\left(1-4 \sigma+4 \sigma^{2}-\sigma^{3}\right)\right] H^{2} k^{2}
\end{aligned}
$$

R.S. acknowledges the financial support from the Egyptian Government under the Postdoctoral Research Program. The work of W.M.M. was partially supported by Ruhr-Universität Bochum through the Framework of the HGF Impulse and Networking Fund/FZ-Jülich (Project Number: S080200W). W.M.M. also thanks Professor R. Schlickeiser for his hospitality.

\section{References}

1. H.R. Miller, P.J. Witta, Active Galactic Nuclei (SpringerVerlag, Berlin, 1987), p. 202; P. Goldreich, W.H. Julian, Astrophys. J. 157, 869 (1969); F.C. Michel, Rev. Mod. Phys. 54, 1 (1982); R. Schlickeiser, P.K. Shukla, Astrophys. J. Lett. 599, L57 (2003)
2. A.P. Lightman, Astrophys. J. 253, 842 (1982)

3. M.L. Burns, R.V.E. Lovelace, Astrophys. J. 262, 87 (1982)

4. A.P. Lightman, A.A. Zdziarski, Astrophys. J. 319, 643 (1987)

5. A.A. Zdziarski, Astrophys. J. 335, 786 (1987)

6. P.K. Shukla, N.N. Rao, M.Y. Yu, N.L. Tsintsadze, Phys. Rep. 138, 1 (1986)

7. V. Berezhiani, D.D. Tskhakaya, P.K. Shukla, Phys. Rev. A 46, 6608 (1992)

8. P. Helander, D.J. Ward, Phys. Rev. Lett. 90, 135004 (2003)

9. E. Tandberg-Hansen, A.G. Emslie, The Physics of Solar Flares (Cambridge University Press, Cambridge, 1988), p. 124 
10. V. Tsytovich, C.B. Wharton, Comments Plasma Phys. Contr. Fusion 4, 91 (1978)

11. R. Bharuthram, Astrophys. Space Sci. 189, 213 (1992)

12. F. Verheest, M.A. Hellberg, G.J. Gray, R.L. Mace, Astrophys. Space Sci. 239, 125 (1995); I. Kourakis, F. Verheest, N. Cramer, Phys. Plasmas 14, 022306 (2007)

13. S.I. Popel, S.V. Vladimirov, P.K. Shukla, Phys. Plasmas 2, $716(1995)$

14. M.Y. Yu, P.K. Shukla, L. Stenflo, Astrophys. J. Lett. 309, L63 (1986)

15. Y.N. Nejoh, Phys. Plasmas 3, 1447 (1996)

16. A. Mushtaq, H.A. Shah, Phys. Plasmas 12, 072306 (2005)

17. N. Shukla, P.K. Shukla, Phys. Lett. A 362, 221 (2007)

18. A. Esfandyari-Kalejahi, I. Kourakis, P.K. Shukla, J. Phys. A 39, 13817 (2006); I. Kourakis, A. Esfandyari-Kalejahi, M. Mehdipoor, P.K. Shukla, Phys. Plasmas 13, 052117 (2006)

19. A. Luque, H. Schamel, R. Fedele, Phys. Lett. A 324, 185 (2004); D. Jovanovic, R. Fedele, Phys. Lett. A 364, 304 (2007)

20. F. Haas, G. Manfredi, M. Feix, Phys. Rev. E 62, 2763 (2000); F. Haas, G. Manfredi, J. Goedert, Phys. Rev. E 64, 026413 (2001); F. Haas, L.G. Garcia, J. Goedert, G. Manfredi, Phys. Plasmas 10, 3858 (2003)

21. P.K. Shukla, B. Eliasson, Phys. Rev. Lett. 96, 245001 (2006); P.K. Shukla, B. Eliasson, New J. Phys. 9, 98 (2007)

22. W.M. Moslem, P.K. Shukla, S. Ali, R. Schlickeiser, Phys. Plasmas 14, 042107 (2007); W.M. Moslem, S. Ali, P.K. Shukla, X.Y. Tang, G. Rowlands, Phys. Plasmas 14, 082308 (2007)

23. P.K. Shukla, S. Ali, Phys. Plasmas 12, 114502 (2005); S. Ali, P.K. Shukla, Phys. Plasmas 13, 022313 (2006)
24. S. Ali, W.M. Moslem, P.K. Shukla, R. Schlickeiser, Phys. Plasmas 14, 082307 (2007); K. Roy, A.P. Misra, P. Chatterjee, Phys. Plasmas 15, 032310 (2008)

25. B. Sahu, R. Roychowdhury, Phys. Plasmas 14, 012304 (2007)

26. A. Mushtaq, S.A. Khan, Phys. Plasmas 14, 052307 (2007)

27. S. Ali, W.M. Moslem, I. Kourakis, P.K. Shukla, New J. Phys. 10, 023007 (2008)

28. W. Masood, A.M. Mirza, M. Hanif, Phys. Plasmas 15, 072106 (2008)

29. A.P. Misra, A. Roychowdhury, Phys. Plasmas 13, 072305 (2006)

30. A.P. Misra, P.K. Shukla, C. Bhowmik, Phys. Plasmas 14, 082309 (2007)

31. A.P. Misra, P.K. Shukla, Phys. Plasmas 14, 082312 (2007)

32. C. Bhowmik, A.P. Misra, P.K. Shukla, Phys. Plasmas 14, 122107 (2007)

33. A.P. Misra, P.K. Shukla, Phys. Plasmas 15, 052105 (2008)

34. T. Taniuti, N. Yajima, J. Math. Phys. 10, 1369 (1969); N. Asano, T. Taniuti, N. Yajima, J. Math. Phys. 10, 2020 (1969); T. Kawahara, J. Phys. Soc. Jpn 35, 1537 (1973); P.K. Shukla, A.A. Mamun, New J. Phys. 5, 17 (2003)

35. X. Jukui, Lang He, Phys. Plasmas 10, 339 (2003)

36. X. Jukui, Phys. Plasmas 11, 1860 (2004)

37. M. Remoissenet, Waves Called Solitons (Springer-Verlag, Berlin, 1996); T. Dauxois, M. Peyrard, Physics of Solitons (Cambridge University Press, Cambridge, 2005)

38. R. Fedele, Phys. Scr. 65, 502 (2002); R. Fedele, H. Schamel, Eur. Phys. J. B 27, 313 (2002)

39. I. Kourakis, P.K. Shukla, Nonlinear Processes Geophys. 12, 407 (2005); I. Kourakis, P.K. Shukla, J. Plasma Phys. $\mathbf{7 1}, 185(2005)$ 